\title{
GESTÃO DE RECURSOS HUMANOS EM PME'S DE ELEVADA TECNOLOGIA
}

\author{
Ana Veloso ${ }^{1}$ \\ José Keating ${ }^{2}$
}

Resumo: Com base nos resultados de três estudos de caso conduzidos em PME do sector das novas tecnologias, procura-se contribuir para a clarificação do(s) processo(s) pelo(s) quais a GRH tem impacto numa organização.

Os três estudos de caso tiveram como objectivos: Caracterizar a gestão de recursos humanos e identificar as práticas utilizadas na organização; Perceber os indicadores utilizados na tomada de decisão para a adopção de novas práticas; Explorar a percepção de impacto da gestão de recursos humanos e/ou das práticas no desenvolvimento organizacional, quer pelos decisores, quer pelos colaboradores da organização, alvos dessa mesma GRH.

Verificámos que o processo de implementação das práticas de GRH em A e C é incremental e resultante de processos informais de benchmarking, enquanto que em $\mathrm{B}$ foi desenhado um pacote de práticas, importadas de multinacionais, que constituiu, desde a fundação da organização, o seu reportório base e formal. A percepção dos colaboradores e dos decisores da importância da GRH para a performance da empresa é diferenciada nas três organizações: em A, atribui-se à GRH um valor instrumental de criação de valor muito claro e as perspectivas de colaboradores e dirigentes são consonantes; em $\mathrm{B}$, a percepção do valor é diferenciada: para os dirigentes, a GRH é uma forma de motivação dos colaboradores garantindo maior eficiência, mas também uma forma de controlo formal; para os colaboradores, uma função essencialmente administrativa. Em $\mathrm{C}$, a função, numa fase de menor amadurecimento, é percepcionada como uma função essencialmente administrativa, mas reconhece-se, quer pelos colaboradores, quer pelos decisores, um potencial essencial ao desenvolvimento futuro da organização. Identificaram-se como factores condicionantes do impacto das práticas de GRH, a nível externo, o mercado (clientes e concorrentes) e, a nível interno, a organização do trabalho e coerência interna do sistema GRH, em termos da harmonização das práticas entre si e com a estratégia da organização.

Os dados resultantes da comparação entre casos evidenciaram alguns aspectos relevantes e inesperados para esta investigação. A natureza da função, GRH é caracterizada como uma função com uma forte componente negocial e de gestão de conflitos. A percepção da utilidade altera-se em função do grau de especializa-

\footnotetext{
${ }^{1}$ Departamento de Psicologia, Universidade do Minho, Braga.

2 Departamento de Psicologia, Universidade do Minho, Braga.

PSICOLOGIA, Vol. XXII (1), 2008, Edições Colibri, Lisboa, pp. 35-58.
} 
ção da função GRH: é maior em A e C, menor em B. Estes resultados sugerem que práticas de GRH formais podem produzir diferentes resultados em termos sociais e que o envolvimento, a compreensão e aceitação das práticas podem ser um prócêsso desenvolvimental. Por outro lado, para a percepção da utilidade da função comtribui, para além destes aspectos, o grau da sua especialização e a coerência do sistema interno de GRH com a estratégia da organização.

Palavras-chave: gestão de recursos humanos, performance organizacional, estudos caso.

Human resource management in high technology sme's (Abstract): The authors examine Human Resource Management (HRM) practices using data from three case studies of high technology entrepreneurial sme's in Portugal and the implementation process and impact on organizational performance that these practices have.

The objectives of the study were to: (1) Describe HRM and identify HRM practices used in the organization; (2) Understand how decisions were made concerning adoption of new HRM practices and; (3) Explore the perception of the primary decisions makers and employees with the organization and their acceptance of HRM systems and practices.

We verify that development of HRM practices were incrementally implemented as problems arose in A, whereas in B they were implemented in a complete set from the beginning. General agreement throughout the organization of the importance of the HRM function. Recognition of the HRM impact on organizational performance are explained differently in each organization: In organization A, HRM function adds value to organization through customers' perceptions of employees' satisfaction; In organization B, HRM motivates workers, which is an expected outcome of HRM practices, and should increase organizational efficiency (i.e. "better work in fewer hours"). In organization C, on a different stage of maturity, HRM function is mainly an administrative function but employees and main decisors recognise HRM potential value, by, for future organization development. The factors who in some way limit the HRM practices impact are, at external level, the market (clients and competitors) and at internal level, work organization and the internal coherence of HRM systems, specially the internal harmonization of the practices and with the organizational strategy

The data produced with case studies comparison are unexpected and relevant. The HRM nature is characterized as a function of strong negotiable component and as a management of conflicts. The perception of utility changed in function of the specialization degree of the HRM function: higher on organizations A and C, lower at organization B. These results suggest that: Formal HRM practices (i.e. those which are "prescribed" and "official") that are similar across organizations can yield different social results. Trust-building practices are an important element in the process through which HRM impacts organizational performance. HRM has to implement a developmental process through which workers can understand and accept HRM practices to have a sustained impact on organizational performance.

Keywords: human resources management, organizational performance, case studies. 


\section{Introdução}

Existe um largo consenso de que a gestão de recursos humanos (GRH) tem impacto na performance organizacional (Huselid, 1995; McDuffie, 1995), reconhecendo-se, no caso particular das pequenas e médias empresas (PME), como sendo um aspecto determinante para o seu crescimento (Cassell, Nadin, Gray \& Clegg, 2002; Heneman, Tansky \& Camp, 2000). Por exemplo, as decisões ao nível da GRH nas fases iniciais de vida de uma pequena e média empresa (PME) têm um profundo impacto no sucesso futuro do negócio e estas opções evoluem e mudam ao longo do tempo e do desenvolvimento organizacional (Cardon \& Stevens, 2004, p. 320). Apesar de alguns autores defenderem que a GRH não é diferente em PME ou em grandes empresas, investigações recentes sugerem que algumas diferenças podem ser encontradas na forma como as pessoas são geridas em PME, nomeadamente em aspectos como o processo de desenvolvimento das políticas e sistema em GRH, a identificação, opção e introdução de práticas de GRH e o grau de formalização que lhes é atribuído (Cardon \& Stevens, 2004; Kotey \& Slader, 2005).

A investigação realizada neste domínio tem-se centrado com especial ênfase na avaliação do impacto da GRH na performance organizacional. Inicialmente, a pesquisa centrou-se numa relação causal de impacto, de que são exemplos os trabalhos de Huselid (1995) e MacDuffie (1995).

Contudo, outras perspectivas surgiram principalmente pela crítica centrada nas limitações metodológicas encontradas nas investigações que suportam a conclusão de que a GRH tem impacto na performance organizacional. Dúvidas são suscitadas quanto à prova irrefutável da relação causal entre GRH e performance organizacional (Ferris et al., 1998) e são sugeridas contaminações entre o investigador e o seu objecto de investigação nomeadamente pelo facto de "... o papel do investigador fosse procurar evidência para uma relação largamente expectável (e mesmo desejada) (Wall \& Wood, 2005, p. 432).

As questões metodológicas que surgem nesta área de investigação são inúmeras e decorrem, em grande medida, do desenvolvimento conceptual dos objectos em avaliação, ou seja, a GRH, a performance organizacional e a própria relação GRH e performance organizacional (Guest, 2001).

A gestão de recursos humanos e o seu impacto na performance organizacional

A definição do que é a função gestão de recursos humanos remete-nos para diferentes conteúdos (Legge, 1995, p. 2-9): (1) regulador das relações de emprego, assumindo que as metas organizacionais, bem como 
os meios necessários à sua realização são sujeitos a negociação; (2) apoio da gestão para atingir as suas metas através do esforço dos colaboradores, sendo a relação estabelecida uma relação de exploração, em contraposição com uma função de regulação; (3) função menor, atribuída àqueles a quem se quer castigar, com reduzida capacidade de intervenção porque suportada num corpo teórico inconsistente e traduzido por um conjunto avulso de técnicas sem credibilidade, sendo percepcionada como um serviço e não como um factor de produção; (4) seleccionar, desenvolver, recompensar e dirigir colaboradores, garantindo que estes obtêm satisfação e "dão o seu melhor" no exercício das suas funções e, assim, permitindo que a organização atinja os seus objectivos.

Em termos operacionais, esta última perspectiva é aquela que mais se tem reflectido nos desenhos experimentais na abordagem da relação de GRH-performance organizacional. Por exemplo, Wood e Wall (2002) definem GRH como o termo utilizado para designar as actividades organizacionais relativas ao recrutamento, desenvolvimento e gestão de colaboradores. Contudo, esta perspectiva coloca também algumas dificuldades e limitações, nomeadamente, na identificação e no número de práticas a eleger e dos indicadores de performance a adoptar.

Alguns investigadores optaram por realizar a avaliação focalizada no efeito de uma prática isolada. Por exemplo, em estudos relativos à selecção e à formação, encontramos, frequentemente, estas práticas correlacionadas quer com a produtividade, quer com a performance organizacional (Youndt et al., 1996). Esta opção tem subjacente a ideia de que o efeito das práticas é aditivo, contrariando a perspectiva do efeito sinergético decorrente da harmonização dos sistemas internos da organização (Becker \& Gerhart, 1996; Marchington \& Grugulis, 2000). No entanto, constata-se a dificuldade de conseguir circunscrever claramente o efeito de uma prática de gestão de recursos humanos na performance da empresa, existindo a possibilidade, não definitivamente refutada, de que esta reflectirá o efeito conjunto de outras práticas adoptadas simultaneamente (Huselid, 1995; Delaney \& Huselid, 1996). Assim, assistiu-se, neste campo de investigação, à transferência do focus experimental do efeito de uma prática isolada de GRH para um conjunto de práticas, destacando-se as investigações de MacDuffie (1995) e Huselid (1995). Estes investigadores evidenciaram um efeito sinergético de um conjunto de práticas de gestão de recursos humanos denominadas por práticas de trabalho de elevada performance (PTEP). Esta denominação decorre do facto de se esperar que a sua utilização possa desenvolver a performance organizacional e enquadra-se na perspectiva de que a GRH produz uma vantagem estratégica para a organização, porque não pode ser replicada, dado o carácter único do conhecimento desenvolvido internamente na organização (Wright \& Gradner, 2000). Esta perspec- 
tiva, decorrente de uma abordagem contingencial, contrasta com a ideia universalista da GRH que defende a necessidade de identificar o conjunto de práticas - as boas práticas - que produzirão na organização sempre o mesmo efeito, independentemente do contexto em que se operacionalizam (Pfeffer, 1994).

A referência a estas práticas (boas práticas e/ou de elevado envolvimento) associa-lhes objectivos de aumento da motivação e das competências dos colaboradores, bem como oportunidades de aplicação de conhecimentos. A sua operacionalização, contudo, não é comum a todas as organizações. Os próprios investigadores utilizam conjuntos diferentes de práticas nas suas investigações, o que constitui um grande obstáculo à acumulação de dados e à generalização das conclusões (Becker \& Gerhart, 1996; Guest, 2001; Marchington \& Grugulis, 2000; Wood, 1999). A questão que se coloca aqui não é a discussão do efeito das práticas de elevada performance ou boas práticas, que se reconhece como evidente, mas sim se existe um conjunto único de práticas (abordagem universalista) ou se este deve ser constituído a partir de um conjunto vasto de práticas, consoante as necessidades da organização (abordagem contingencial). Aliás, Wood (1999) refere que encontramos um esforço de conciliação destas duas perspectivas, em Huselid e Becker, quando estes propõem como principal factor diferenciador na adopção das práticas de elevada performance ou boas práticas o processo de implementação e de adopção das práticas.

Existe algum consenso de que as práticas de elevada performance são aquelas que envolvem elevado compromisso e envolvimento (Bélanguer, 2000; Wood, 1999). Nos diferentes estudos sobre este assunto (Bélanguer, 2000; Becker \& Huselid, 1999; Delaney \& Huselid, 1996; Delery \& Doty, 1996; Huselid, 1995; MacDuffie, 1995; Patterson et al., 1997; Richard \& Johnson, 2001) encontramos diferentes práticas que se agrupam em grandes vectores: motivacional (compensação, gestão de carreira), participação (equipas autónomas; mecanismos de participação, presença de sindicatos, job design) e desenvolvimento (formação, avaliação de desempenho centrada na performance), reflectindo, como já foi referido, a utilização de uma grande diversidade de práticas e evidenciando a dificuldade de justificar a opção por um único conjunto de práticas.

A crítica fundamental relativamente às práticas de elevado envolvimento é de que estas promovem o maior esforço dos trabalhadores (mais tempo de trabalho, por exemplo), não se verificando, contudo, alterações ao nível da autonomia e desenho das funções, pelo que será natural terem impacto na produtividade e performance organizacional (Bélanguer, 2000).

A avaliação do impacto das práticas de elevado envolvimento introduziu neste debate questões como (1) fit, ou seja, adequação de um conjunto de práticas à organização (Wood, 1999) e não necessariamente a identifica- 
ção de um conjunto rígido de boas práticas e (2) a adopção de outros indicadores de impacto que não apenas os financeiros, como, por exemplo, a motivação e a satisfação dos colaboradores.

A perspectiva de que a GRH poderá ter impacto na performance organizacional porque promove o contributo dos colaboradores, motivando-os e envolvendo-os, introduz uma mudança no papel dos colaboradores neste processo. Mais do que objecto, sobre o qual as práticas produzem um efeito, os colaboradores são um meio, através do qual as práticas produzem efeito na organização. Apenas ganhando o compromisso dos colaboradores é possível atingir as metas organizacionais (Guest, 1999). Esta mudança de perspectiva contribuiu para que, nas metodologias desta área de investigação, se diversificasse as fontes de informação e se passasse a considerar também como fonte relevante os colaboradores, nomeadamente, a recolha de informação sobre as suas expectativas e percepções sobre a GRH. Guest (1999), defendendo que é necessário considerar a perspectiva dos colaboradores sobre a GRH, sugere que, ao se incluir a sua perspectiva, se preencham as seguintes condições na metodologia de investigação: (1) definição de critérios que reflictam a força do sentimento, o sentido de justiça ou injustiça e as percepções de ganho e perda; (2) se efectuem investigações que permitam e/ou contribuam para a clarificação da relação entre GRH e performance organizacional, e especificamente se possa determinar se o impacto se deve a maior intensificação do trabalho, por exemplo, ou a maiores níveis de motivação, utilização de competências e envolvimento e (3) se considerem outros protagonistas, para além dos gestores, como sejam os accionistas e os sindicatos. Em termos gerais, outros parceiros activos.

Outras sugestões têm sido apresentadas relativamente à metodologia de investigação para avaliar o impacto da GRH na performance da empresa. Wright e Gardner (2000) referem que tem sido dada reduzida atenção à exploração dos processos pelos quais a GRH tem impacto na organização, salientando a falta de consenso entre os investigadores, sobre este mesmo impacto. Sugerem a necessidade de focalização de estudos intra-indústria, realizados ao nível de fábrica ou negócio e de medidas fiáveis das práticas de GRH. Relativamente a este último ponto, aconselham a inclusão de relatos dos trabalhadores sobre as práticas de GRH. Por último, sugerem a adopção de investigação qualitativa e longitudinal como uma forma de desenvolver conceptualmente este domínio.

Em síntese, e sublinhando os aspectos que orientaram o desenho da nossa investigação, quando se procura explicar o impacto da GRH na performance organizacional, é importante (1) descrever e compreender os procêssos através dos quais este impacto ocorre, (2) utilizar indicadores que reflictam de forma rigorosa as acções da GRH e (3) procurar outras fontes de 
informação, como, por exemplo, os colaboradores (Becker \& Gerhart, 1996; Bélanger, 2000; Godard \& Delaney, 2000; Guest, 1999; Wood, 1999).

É intenção dos autores contribuir para a clarificação do(s) processo(s) pelo(s) quais a GRH tem impacto numa organização, apresentando-se de seguida três estudos de caso conduzidos em PME do sector das novas tecnologias. Estes estudos de caso tiveram como objectivos:

- Caracterizar a gestão de recursos humanos e identificar as práticas utilizadas na organização;

- Perceber os indicadores utilizados na tomada de decisão para a adopção de novas práticas;

- Explorar a percepção de impacto da gestão de recursos humanos e/ou das práticas no desenvolvimento organizacional, quer pelos decisores, quer pelos colaboradores da organização, alvos dessa mesma GRH.

\section{Metodologia}

\section{O estudo de caso como opção metodológica}

$\mathrm{O}$ estudo de caso, como metodologia a adoptar na análise das relações entre gestão de recursos humanos e performance organizacional, tem sido sugerido por vários autores (Guest, 1987, 2001; Wright \& Gardner, 2000). Esta sugestão vem ao encontro de necessidades de desenvolvimento conceptual com que se debate a GRH. Enquanto abordagem distinta, a GRH é uma disciplina em processo de definição e clarificação conceptual e utiliza como fontes de informação privilegiadas, para responder às suas necessidades de desenvolvimento, (1) a investigação em Psicologia Organizacional em domínios específicos como job design, definição de objectivos, etc., (2) o estudo de organizações de sucesso (como, por exemplo, o trabalho de Peters e Waterman (1987)) e (3) os estudos de caso (Guest, 1987).

Esta metodologia poderá, neste caso específico, contribuir para uma maior compreensão do processo pelo qual as práticas de gestão de recursos humanos operam, interagem, combinam e entram em conflito, bem como clarificar a relação de causalidade entre as práticas de gestão de recursos humanos e a performance organizacional. A título de exemplo, Wright e Gardner (2000) apontam a utilização de estudos de caso como uma opção necessária quando se pretende descrever de forma eficaz as diferenças entre políticas de GRH defendidas e a sua implementação.

Por outro lado, apesar de se encontrar consistentemente na literatura estudos empíricos que relacionam a gestão de recursos humanos e a performance organizacional, são escassos aqueles que evidenciam o processo pelo qual as organizações podem gerir as suas pessoas de forma bem sucedida (Becker \& Huselid 1999). 
Foram realizados três estudos de caso em três médias empresas no sector das novas tecnologias. A opção por organizações desta dimensão prende-se com o facto de não existir investigação substancial sobre a GRH em PME (pequenas e médias empresas), especificamente na identificação das práticas utilizadas, ainda que a GRH seja um aspecto essencial para estas empresas (Cassell; Nadin, Gray \& Clegg, 2002; Rowden, 1995) e no contexto português ser este o tipo prevalecente de organizações (Keating et al., 1999).

A selecção de casos, segundo Yin (1994), deverá considerar a possibilidade de (1) predizer resultados similares, ou seja, uma replicação literal ou (2) produzir resultados contrastantes, mas por razões preditíveis, isto é, uma replicação teórica. A opção dos autores contemplou a primeira, replicação literal, pelo que se procurou definir, tal como Yin (1994) refere, as condições segundo as quais um fenómeno particular ocorrerá (uma replicação literal). Assim, na escolha das organizações pretendeu-se realizar um controlo de factores, como a dimensão, o número de colaboradores, a antiguidade da organização e o sector de actividade.

Tabela 1. Características das organizações estudadas

\begin{tabular}{|c|c|c|c|}
\hline & Organização A & Organização B & Organização C \\
\hline $\begin{array}{l}\text { Dimensão } \\
\text { (Número } \\
\text { colaboradores) }\end{array}$ & 100 & 250 & 120 \\
\hline Equipa Dirigente & $\begin{array}{l}\text { Administração } \\
\text { (DRH) }\end{array}$ & $\begin{array}{l}\text { Administração } \\
\text { (Técnico) }\end{array}$ & $\begin{array}{l}\text { Administração } \\
\text { (RH e Técnico) }\end{array}$ \\
\hline Negócio & $\begin{array}{l}\text { Produção de } \\
\text { Software }\end{array}$ & $\begin{array}{l}\text { Consultoria e } \\
\text { Software }\end{array}$ & $\begin{array}{l}\text { Consultoria e } \\
\text { Software }\end{array}$ \\
\hline Antiguidade & 10 Anos & 3 Anos & 7 Anos \\
\hline Colaboradores & $\begin{array}{l}\text { Elevado nível } \\
\text { habilitacional. } \\
\text { Baixo Turnover }\end{array}$ & $\begin{array}{l}\text { Elevado nível } \\
\text { habilitacional. } \\
\text { Baixo Turnover }\end{array}$ & $\begin{array}{l}\text { Elevado nível } \\
\text { habilitacional. } \\
\text { Baixo Turnover }\end{array}$ \\
\hline $\begin{array}{l}\text { Estrutura } \\
\text { Organizacional }\end{array}$ & Matriz & Matriz & Matriz \\
\hline
\end{tabular}

Os três estudos de caso foram realizados sequencialmente. Procedeu-se à análise individual e detalhada de cada um dos casos individualmente (análise intra casos) passando-se posteriormente à análise entre casos. 
Os dois primeiros estudos de caso, exploratórios, permitiram-nos identificar padrões únicos e um conhecimento detalhado que facilitou posteriormente a análise entre casos. Esta última, a análise entre casos, procura identificar padrões comuns, ultrapassando impressões iniciais e descrições de casos únicos.

A análise comparativa de casos aumenta a probabilidade de novos contributos para a teoria com base na adequação dos dados recolhidos. Potencia também a probabilidade de identificar novos elementos que possam existir nos dados. A utilização das duas formas de análise fortalece a validade e fidelidade dos resultados das investigações (Rowden, 1995, p. 359).

Este conjunto de três estudos de caso que aqui apresentamos não foi realizado segundo uma perspectiva de amostragem mas sim de replicação, isto é, quando realizamos múltiplos estudos de caso, devemos, na opinião de Yin (1994), considerá-los como múltiplas experiências e não como diferentes sujeitos a responder a um mesmo questionário.

A abordagem de replicação utilizando um desenho de múltiplos estudos de caso contempla as seguintes fases: (1) desenvolvimento da teoria; (2) selecção de casos e definição de medidas de avaliação, que são aspectos essenciais no desenho do protocolo de recolha de dados; (3) condução dos estudos de caso individuais e sua análise (a sucessão dos estudos de caso individuais é uma importante fonte de replicação interna das conclusões dos estudos de caso individuais anteriores); (4) elaboração do relatório integrando (a) as conclusões dos estudos de caso individuais e (b) a comparação intercasos. Este relatório apresenta como e porquê as proposições foram (ou não) demonstradas, explicitando a sua contribuição para a teoria, quer pela sua modificação, quer pela sua confirmação.

\section{Plano de investigação}

Foi utilizado o mesmo protocolo nos três estudos de caso. Realizaram-se entrevistas a todos os níveis hierárquicos das organizações, desde os fundadores e administradores a colaboradores, com uma presença mínima na organização de 6 meses. Incluiu-se no grupo "colaboradores" todos aqueles que não exercem funções de direcção.

Procurou-se, assim, obter informações de colaboradores com diferentes anos de antiguidade na empresa e que abrangeram colaboradores presentes na organização desde a sua fundação até à data de realização do estudo.

A estrutura do guião das entrevistas realizadas está reflectida no template adoptado para análise, como explicaremos posteriormente (ver Tabela 3). 


\section{Os dois estudos de caso exploratórios}

Foram realizadas, numa primeira fase, 17 entrevistas nos dois primeiros estudos de caso (ver Tabela 2), a que se seguiram mais 5 entrevistas numa segunda fase e com um intervalo de tempo não inferior a seis meses. Estas segundas entrevistas foram realizadas aos mesmos indivíduos entrevistados nas entrevistas iniciais e estão discriminadas na Tabela 2. No caso da organização A, o número de entrevistas foi superior ao das restantes organizações, devido à natureza exploratória do estudo de caso. As entrevistas foram gravadas e integralmente transcritas. Em complemento, consultou-se documentação disponibilizada pela empresa.

\section{O terceiro estudo de caso}

No terceiro estudo de caso adoptou-se a mesma estratégia de recolha de informação. Foram realizadas entrevistas a todos os níveis da hierarquia e anos de antiguidade, tendo-se realizado uma segunda entrevista à técnica de RH, num total de 8 entrevistas. O guião de entrevista foi também o mesmo.

Tabela 2. Entrevistas realizadas.

\begin{tabular}{|c|c|c|c|c|c|c|}
\hline & \multicolumn{2}{|c|}{ Organização A } & \multicolumn{2}{|c|}{ Organização B } & \multicolumn{2}{|c|}{ Organização C } \\
\hline & \multicolumn{2}{|c|}{ Entrevistas } & \multicolumn{2}{|c|}{ Entrevistas } & \multicolumn{2}{|c|}{ Entrevistas } \\
\hline & 1. ${ }^{\text {as }}$ & $2 .^{\text {as }}$ & $1 .^{\text {as }}$ & $2 .^{\text {as }}$ & $1 .^{\text {as }}$ & $2 .^{\text {as }}$ \\
\hline Administradores & 3 & 1 & 2 & & 2 & \\
\hline Director & 1 & & 2 & & 2 & \\
\hline Director RH & 1 & 1 & 1 & 1 & & \\
\hline Técnico de RH & 1 & 1 & 1 & 1 & 1 & 1 \\
\hline Técnicos & 2 & 1 & 4 & & 2 & \\
\hline Total & 7 & 3 & 10 & 2 & 7 & 1 \\
\hline
\end{tabular}

Metodologia de análise das entrevistas

A análise dos dados recolhidos foi realizada com recurso à template analysis (King, 1998), em que o texto de transcrição das entrevistas tem um especial relevo.

Esta análise recorre à utilização de um conjunto de códigos (o template) produzido pelo investigador que representa temas identificados nos 
seus dados (King, 1998, p. 119). Os dados apresentam-se no formato de texto e resultam frequentemente da transcrição de entrevistas e focus groups, observação participante, diários de investigação, documentos organizacionais, etc. No caso desta investigação, os dados recolhidos resultaram da transcrição integral das entrevistas realizadas.

Esta metodologia de análise é dotada de grande flexibilidade porque permite a adaptação do template ao longo da análise dos dados, ainda que exija ao investigador uma abordagem bem estruturada no manuseamento dos dados, para que, no final, consiga produzir um estudo inteligível.

\section{Definição de códigos}

$\mathrm{Na}$ análise dos textos, o investigador identifica partes relacionadas com temas ou assuntos importantes para a sua interpretação, a que atribui um código. Os códigos podem ser interpretativos ou descritivos, sendo estes últimos os que exigem menor avaliação por parte do investigador sobre o significado do sentido do discurso do entrevistado. $\mathrm{Na}$ análise das entrevistas dos estudos de caso, os códigos atribuídos prenderam-se com a identificação no texto de referências às práticas de GRH, às percepções dos entrevistados sobre o seu impacto, independentemente da forma como esse impacto era traduzido (experiências próprias, resultados da empresa, acontecimentos significativos, etc.).

\section{Desenvolvimento do template}

O desenvolvimento do template não é um processo isolado da sua utilização na análise do texto, ainda que esta se inicie por identificar previamente um conjunto de alguns códigos (pré-definidos) que orientam inicialmente a análise. Um template é utilizado na análise do texto durante o processo de codificação e em simultâneo sujeito a revisão e reconstrução. Segundo King (1998), um ponto de partida para a construção do template inicial é o guião da entrevista. Este guião reflecte um trabalho anterior, do investigador, de pesquisa que define o enquadramento conceptual e teórico da investigação, e recebe os contributos da experiência do investigador e de investigação exploratória prévia. As questões incluídas no guião da entrevista poderão, assim, ser utilizados como códigos iniciais na análise dos dados.

Nesta investigação, o desenvolvimento do template cumpriu estes procedimentos. Alguns códigos foram identificados com base na revisão da literatura e com os objectivos da investigação, tendo-se definido um template que se caracterizou por ser abrangente com um conjunto sucinto de códigos. A hierarquização dos códigos foi reduzida, esperando-se que se verificasse a integração de novos códigos e/ou a revisão de outros inicialmente identificados. Esta expectativa resultou do facto de se estarem, primeiramente, a conduzir estudos de caso exploratórios, que, pela sua nature- 
za dinâmica, propiciam a identificação de novos aspectos num prócesso dinâmico de análise e de construção do conhecimento. Também, não houve necessidade de utilizar códigos paralelos face à riqueza dos dados recolhidos, em termos de qualidade e quantidade.

Especificando, apresentamos de seguida a evolução do template utilizado nos estudos de caso (ver Tabela 3). A identificação das práticas de GRH existentes nas organizações foi realizada pelos entrevistados de uma forma muito pormenorizada. Assim, surgiram no corpo do texto analisado, no conjunto dos três estudos de caso, referências recorrentes a temas que receberam os seguintes códigos: recrutamento e selecção, integração, formação, avaliação de desempenho, gestão de carreiras/progressão, prémios e remuneração e saídas.

$\mathrm{Na}$ avaliação de impacto das práticas de GRH foram considerados novos códigos que tornaram mais especializada a análise, tendo-se incluído os códigos participação, motivação e comunicação.

No decorrer da análise de dados foi incluído também um código relativo a Mudança, no caso da Organização A, e, no caso da Organização B, um outro referente à Organização do Trabalho. No período de realização das entrevistas estava a decorrer um processo de reestruturação importante da organização, pelo que este tema surgiu de forma recorrente no corpo das entrevistas. Tendo implicações importantes ao nível da gestão de recursos humanos, em especial nas percepções dos colaboradores sobre a sua eficácia, considerou-se relevante a sua inclusão no template.

Tabela 3. Template inicial utilizado no estudo de caso da Organização A e o seu desenvolvimento para os estudos de caso seguintes

\begin{tabular}{lll}
\hline Template inicial & Template final & Template final \\
Organização A & Organização A & Organização B
\end{tabular}

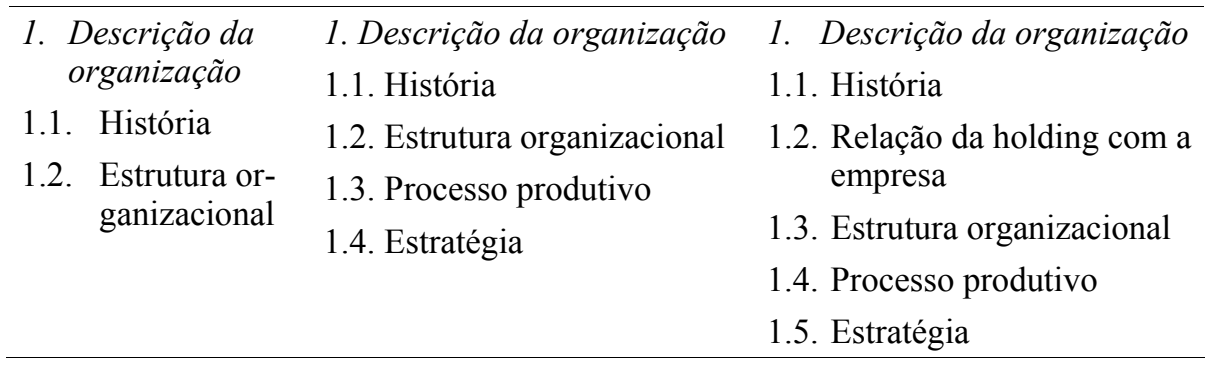




\begin{tabular}{|c|c|c|}
\hline $\begin{array}{l}\text { Template inicial } \\
\text { Organização A }\end{array}$ & $\begin{array}{l}\text { Template final } \\
\text { Organização A }\end{array}$ & $\begin{array}{l}\text { Template final } \\
\text { Organização B }\end{array}$ \\
\hline 2. Papel da GRH & 2. Papel da GRH & 2. Papel da GRH \\
\hline \multirow[t]{9}{*}{$\begin{array}{l}\text { 3. Identificação das } \\
\text { práticas de GRH }\end{array}$} & $\begin{array}{l}\text { 3. Identificação das práticas } \\
\text { de GRH }\end{array}$ & $\begin{array}{l}\text { 3. Identificação das práticas de GRH } \\
\text { 3.1. Recrutamento e seleccão }\end{array}$ \\
\hline & 3.1. Recrutamento e selecção & \\
\hline & 3.2. Integração & 3.2. Integração \\
\hline & 3.3. Formação & 3.3. Formação \\
\hline & $\begin{array}{l}\text { 3.4. Avaliação de } \\
\text { desempenho }\end{array}$ & 3.4. Avaliação de desempenho \\
\hline & 3.5. Gestão de & 3.5. Gestão de carreiras/progressão \\
\hline & carreiras/progressão & 3.6. Prémios e remuneração \\
\hline & 3.6. Prémios e remuneração & \\
\hline & 3.7. Saídas & 3.7. Gestão das Saídas \\
\hline $\begin{array}{l}\text { 4. Processo de } \\
\text { introdução das } \\
\text { práticas de GRH }\end{array}$ & $\begin{array}{l}\text { 4. Processo de introdução } \\
\text { das práticas de GRH }\end{array}$ & $\begin{array}{l}\text { 4. Processo de introdução das } \\
\text { práticas de GRH }\end{array}$ \\
\hline \multirow{10}{*}{$\begin{array}{l}\text { 5. Avaliação de } \\
\text { impacto das } \\
\text { práticas de GRH } \\
\text { 5.1. Identificação } \\
\text { dos indicadores } \\
\text { de impacto }\end{array}$} & $\begin{array}{l}\text { 5. Avaliação de impacto das } \\
\text { práticas de GRH }\end{array}$ & $\begin{array}{l}\text { 5. Avaliação de impacto das } \\
\text { práticas de GRH. }\end{array}$ \\
\hline & 5.1. Identificação dos & 5.1. Identificação dos indicadores de \\
\hline & indicadores de impacto & impacto \\
\hline & 5.1.1 Participação & 5.1.1 Participação \\
\hline & 5.1.2 Motivação & 5.1.2 Motivação \\
\hline & 5.1.3 Comunicação & 5.1.3 Comunicação \\
\hline & & 5.1.4 Cultura \\
\hline & & 5.1.5 Qualidade de vida no trabalho \\
\hline & 6. Processos de mudança & 6. Organização do trabalho \\
\hline & $\begin{array}{l}\text { 6.1. Mudança de estrutura } \\
\text { organizacional }\end{array}$ & $\begin{array}{l}\text { 6.1. A unidade de desenvolvimento } \\
\text { de produto }\end{array}$ \\
\hline
\end{tabular}

\section{Resultados}

Os estudos de caso exploratórios

Ao realizarmos a análise individual dos dois primeiros estudos de caso encontramos uma aparente similaridade nas duas organizações: utilizam os mesmos indicadores de performance para a GRH (volume de vendas; satis- 
fação dos colaboradores), as práticas de GRH adoptadas são formalmente idênticas e possuem objectivos idênticos para a GRH: força de trabalho motivada, satisfeita e produtiva. Contudo, encontramos diferentes resultados das práticas de GRH, em termos qualitativos, nomeadamente quando analisamos as percepções dos colaboradores, que se expressam ao nível do compromisso e empenhamento.

Os dados resultantes da comparação entre casos permitiram evidenciar alguns aspectos relevantes e inesperados para esta investigação, nomeadamente, (1) as diferentes percepções do papel e utilidade da função GRH; (2) o processo de implementação das práticas de GRH e as suas implicações na percepção de impacto da função GRH, (3) factores condicionantes do impacto das práticas de GRH, a nível externo, como, por exemplo, o mercado (clientes e concorrentes) e, a nível interno, a organização do trabalho e coerência interna do sistema GRH e, em especial, o diferente tempo de vida das organizações, que permitem colher distintos resultados de intervenções, após algum tempo de maturação, como, por exemplo, a formação ou a gestão de carreiras.

\section{A natureza e utilidade da função GRH}

Ambas as organizações concordam sobre a importância da GRH e reconhecem que esta tem impacto na performance organizacional. É atribuído à GRH um valor instrumental, sendo utilizada de forma intencional, pelos níveis superiores da hierarquia.

Internamente, os administradores das organizações A e B referem que o mercado externo avalia a estabilidade das organizações pela sua capacidade em manter e mesmo aumentar a sua força de trabalho, bem como pela expressão de satisfação pelos seus colaboradores aos clientes e parceiros, no contexto da sua relação formal e informal de trabalho.

Em termos formais, este impacto é avaliado pela taxa de saídas dos colaboradores e pelo volume de vendas.

As práticas adoptadas por estas organizações têm o objectivo claro de aumentarem o crescimento da organização, em volume de vendas e promoverem a manutenção da força de trabalho, através da retenção de colaboradores (taxa de saídas/ índice de turnover). Assim, verificamos a presença de práticas de GRH que suportam o clima social e motivacional da organização, como sejam a gestão de carreiras, a avaliação de desempenho, bem como de actividades que promovem o espírito de grupo e o envolvimento dos colaboradores na empresa, alvo de particular atenção.

Contudo, o valor intrínseco e a justificação dos efeitos da GRH são diferentes nas duas organizações. Para a organização A, a GRH adiciona valor à organização, através da percepção que os clientes têm do nível de satisfação experimentado pelos seus colaboradores. Para a organização B, colaboradores motivados, um dos resultados esperados das práticas de GRH 
será aumentar a eficiência organizacional, sendo esta traduzida por mais horas de trabalho.

É de notar também que, em termos funcionais, a área da GRH tem diferentes posicionamentos, o que traduz o seu valor relativo na organização. A organização A tem um administrador com a responsabilidade da GRH e uma direcção de Recursos Humanos envolvida nas decisões estratégias. A organização B tem uma técnica de RH que é enquadrada pela direcção administrativo-financeira da empresa e pela DRH do grupo a que pertence.

O processo de implementação das práticas de GRH e as suas implicações na percepção de impacto da função GRH

Outro dos aspectos que se evidenciou na análise entre casos dos dois estudos de caso exploratórios foi o desenvolvimento da função GRH, em especial a introdução de práticas de GRH. Na organização A, o processo foi incremental em resposta a um problema e na organização $\mathrm{B}$, as práticas foram definidas e introduzidas em conjunto, desde a criação da organização. É aqui que reside a principal diferença no que se refere às práticas de elevado envolvimento nestas duas organizações: o processo de introdução das práticas. De facto, as expressões formal e técnica das práticas de GRH são similares nas duas organizações (recrutamento e selecção, gestão de carreiras, avaliação de desempenho, gestão por equipas, etc.), mas o processo de introdução (negociado em A e imposto em B) promoveu diferentes resultados em termos de percepção de utilidade da função GRH, bem como no envolvimento e compromisso com a organização.

Este processo de desenvolvimento da função produziu diferentes resultados nos colaboradores. Por exemplo, a percepção dos colaboradores sobre a GRH é, em A, clara no que se refere à identificação das práticas de GRH existentes e à compreensão dos seus objectivos como estratégicos para a organização. Consideram a função GRH como diferenciada e estratégica.

Os colaboradores da organização B percepcionam a GRH como "burocrática" e sem valor/contributo directo para o seu trabalho, ou seja, uma função administrativa e não estratégica. Para além disso, os colaboradores nas duas organizações apresentam diferentes experiências relativamente à forma como se gere o conflito internamente: enquanto, em $\mathrm{A}$, não existem canais formais de expressão de conflito, incentivando-se a comunicação informal e próxima entre colaboradores, chefias e administração, em $\mathrm{B}$, promove-se a desqualificação do conflito, ainda que haja referência pelos colaboradores de canais formais de expressão de conflitos, traduzindo uma relação hierarquizada com as chefias.

O controlo dos colaboradores é flexível em A, e formal, orientado para os resultados, em B. O exercício diferenciado do controlo surpreende, pois, o conjunto de práticas utilizadas por ambas as organizações e o objec- 
tivo de motivar e manter satisfeita a força de trabalho apontaria para uma forma de controlo flexível, privilegiando a autonomia e a participação (Wall $\&$ Wood, 2005). No caso da organização B, o controlo é bastante rígido e, em algumas áreas da organização, suportado por uma organização de trabalho taylorizada, isto é, especializada.

A filosofia de retenção dos colaboradores é a de manter equipas, na organização A, e de reter colaboradores específicos em B, ou seja, procura-se desenvolver estratégias que mantenham equipas coesas a funcionar em $\mathrm{A}$, enquanto que, em $\mathrm{B}$, nos confrontamos com uma estratégia de retenção centrada no indivíduo.

Por outro lado, ainda que técnica e formalmente ambas as organizações utilizem a GRH para obterem sucesso, sabemos que existem alguns aspectos que condicionam a prossecução deste objectivo. Collins et al. (2002) afirmam que o investimento num conjunto de práticas de GRH poderá ter diferentes resultados, considerando, por exemplo, o grau de harmonização da GRH com os objectivos estratégicos organizacionais, para além de, e na opinião de Bamberger et al. (1989), as especificidades do sector de actividade, o estádio de desenvolvimento da organização, que na sua perspectiva está intimamente associado com o tempo de desenvolvimento da organização, e a estratégia de negócio serem factores a ter também em atenção.

Factores condicionantes do impacto das práticas de GRH

A análise do impacto da GRH na performance organizacional é complexa, especialmente quando se reconhece a influência de outros factores, como, por exemplo, o mercado ou a estratégia de negócio ou mesmo o estado de desenvolvimento da organização, que podem actuar como variáveis moderadoras. Bamberger et al. (1989, pp. 362 e 363) concluíram que, no início da actividade de empresas de elevada tecnologia em Israel, o crescimento da empresa em volume de vendas ou do seu nível de inovação pode resultar mais de políticas e práticas de natureza financeira e de marketing, adoptadas por estas organizações, do que propriamente da GRH, especialmente quando se considera resultados de curto prazo.

De facto, os ciclos produtivos de inovação e gestão são, em organizações de novas tecnologias, mais rápidos do que os ciclos da gestão de pessoas, cujos resultados demoram mais tempo a evidenciarem-se. As intervenções da GRH na organização, nomeadamente no desenvolvimento dos seus colaboradores, através da formação ou da gestão de carreiras, exigem tempo de maturação.

Kotey e Slade (2005, p. 36) afirmam, com base nos resultados da investigação que realizaram e em suporte à teoria dos ciclos de vida, que se verifica uma mudança com o decorrer do tempo de actividade da organização, de estruturas simples com sistemas informais e muito centralizados 
para estruturas funcionais com funções especializadas e maior estandardização e formalização de actividades. Para além disso, referem que, na generalidade dos casos, a adopção de práticas de GRH formais em PME pode também ser explicada pela dimensão da própria organização, ou seja, pelo aumento do número de colaboradores!

Os nossos resultados vão ao encontro do que é apresentado por estes autores. Como já referimos, as duas organizações em análise têm diferentes tempos de vida, a Organização B, 3 anos, e a Organização A, 10 anos.

A título de exemplo e no caso da Organização B, o seu crescimento acelerado foi resultado da integração de pessoas ou grupos já com níveis elevados de competência. O recrutamento e selecção inicial foram focalizados na prócura e escolha de pessoas com perfis elevados de competência e experiência profissional relevante. Deste modo, puderam dar resposta às solicitações dos seus clientes de forma imediata. O seu esforço de desenvolvimento, suportado pela formação e gestão de carreiras, entre outras práticas, após três anos de investimento, poderá começar agora a revelar o seu impacto.

A Organização A, após 10 anos de actividade, com um grande investimento no desenvolvimento e retenção de pessoas, mas com uma politica de recrutamento de recém-licenciados, com elevada qualificação mas reduzida experiência, tem vindo a obter resultados positivos na sua performance e crescimento continuado, em todos os indicadores utilizados para avaliação: volume de vendas, número de clientes e número de colaboradores.

Por outro lado, a Organização B privilegiou o recrutamento de novos colaboradores, para níveis elevados da hierarquia, através de contactos pessoais de alguns dos seus colaboradores (de nível superior, por exemplo, manager), sem contudo, deixar de cumprir os procedimentos previamente formalizados quanto à selecção, ou seja, entrevista, avaliação psicológica, etc. Este procedimento fomentou um compromisso dos colaboradores focalizado primordialmente na chefia da equipa, em detrimento da própria organização.

O resultado da política de recrutamento da Organização A evidencia um compromisso mais centrado na organização do que na figura do líder da equipa e é fruto das suas práticas de recrutamento. Os colaboradores que foram integrados na organização propuseram-se a trabalhar nesta por iniciativa própria (responderam a um anúncio de recrutamento ou fizeram uma candidatura espontânea) e a sua integração foi realizada em torno da organização e não de uma figura individual privilegiada. A Organização A não promove o recrutamento personalizado, ou seja, por contactos pessoais.

Ambas as organizações têm actualmente uma estrutura funcional em matriz. Este modelo de estrutura foi a que a organização B adoptou desde a sua criação, a organização A evoluiu de uma estrutura piramidal e departamentalizada para a estrutura actual. 
Estes dois estudos de caso, se nos focalizarmos no tempo de existência, evidenciam resultados qualitativamente diferentes, considerando apenas a descrição das práticas de GRH existentes e que não se tornam visíveis, se ponderarmos unicamente os resultados de performance e/ou a realização dos objectivos de gestão definidos. É importante considerar, nesta análise, a possibilidade de o impacto das práticas de GRH exigir tempo de maturação para se expressar e que indicadores qualitativos, como o compromisso e o envolvimento com a organização, sejam mais exigentes e finos na determinação desse impacto.

Assim, para além da presença e da diversidade de práticas de GRH na organização, o tempo de vida da organização e os resultados observados em termos de envolvimento e compromisso com a organização passaram a revestir-se de grande importância para esta investigação.

Figura 1. Análise comparativa dos estudos de caso da Organização A e Organização B.

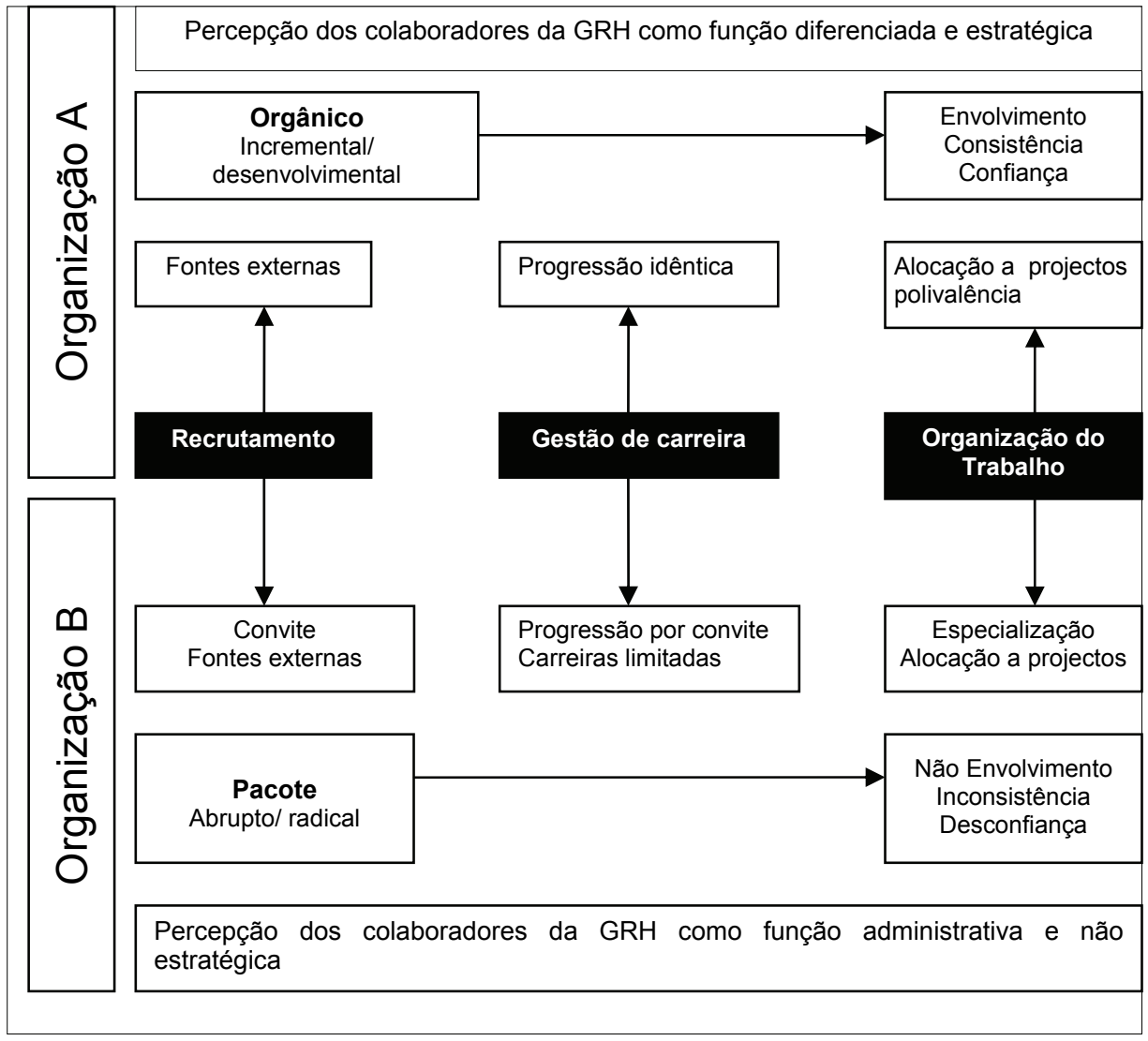




\section{O terceiro estudo de caso}

As principais conclusões, colhidas na análise entre casos que anteriormente apresentámos, influenciaram este terceiro estudo de caso, quer na escolha da organização, quer na maior focalização na recolha de dados.

Escolhemos uma organização do mesmo sector de actividade, com um número de colaboradores e um período de vida próximos da organização A. O nosso focus de análise centrou-se em especial no desenvolvimento da função GRH e na introdução de práticas de GRH, para além dos próprios efeitos que lhe são atribuídos, como sejam o envolvimento e compromisso com a organização.

Constatamos que a GRH é, nesta organização, responsabilidade de um dos cinco administradores e operacionalmente executada por uma técnica. Está prevista a evolução da função para o nível de direcção, proporcionando-lhe maior visibilidade.

A estrutura desta empresa evoluiu, à semelhança da organização A, de uma estrutura hierárquica piramidal e departamentalizada para uma estrutura em matriz e funcional.

As conclusões elaboradas a partir da análise entre casos dos casos da Organização A e Organização B levantaram a questão de se as diferenças encontradas se poderiam explicar também pelo tempo de vida da organização, como referimos anteriormente, já que as práticas encontradas eram semelhantes, em termos técnicos.

Verificamos, neste terceiro estudo de caso, que as práticas de GRH foram introduzidas de forma incremental e em função de necessidades percebidas. Contudo, nesta organização, constatamos uma aceleração do processo, devido ao efeito que os procedimentos das certificações de qualidade, efectuadas devido à exigência de clientes, introduziram.

O conjunto de práticas é consistente com a estratégia de desenvolvimento da organização, na medida em que a introdução das práticas na organização se faz também para dar resposta às novas necessidades que a estratégia organizacional coloca em termos de GRH, como, por exemplo, o esforço de internacionalização. Por outro lado, o número crescente de colaboradores obriga à introdução ou alteração das práticas de GRH.

O grau de sofisticação das práticas utilizadas, ou seja a diversidade de práticas presentes e o seu nível de complexidade, é menor do que na Organização A. Contudo, as perspectivas de desenvolvimento expressas pela organização $C$ vão ao encontro dos níveis da Organização $A$. Um outro aspecto relevante para esta investigação é que encontramos expressões de envolvimento e compromisso, por parte dos colaboradores, idênticos aos da organização A, ou seja, focalizados na organização como um todo. 
Tabela 4. Síntese dos dados na análise entre casos.

\begin{tabular}{|c|c|c|c|}
\hline & Organização A & Organização B & Organização C \\
\hline Dimensão & $\begin{array}{l}100 \\
\text { colaboradores }\end{array}$ & 250 colaboradores & 120 colaboradores \\
\hline Tempo de vida & 10 anos & 3 anos & 7 anos \\
\hline $\begin{array}{l}\text { Desenvol- } \\
\text { vimento }\end{array}$ & $\begin{array}{l}\text { Incremental/ } \\
\text { interno } \\
\text { Integrativo }\end{array}$ & $\begin{array}{l}\text { Programático/ } \\
\text { externo }\end{array}$ & $\begin{array}{l}\text { Incremental/ interno } \\
\text { Integrativo } \\
\text { Externo (certificação da } \\
\text { qualidade) }\end{array}$ \\
\hline $\begin{array}{l}\text { Relação } \\
\text { "práticas GRH/ } \\
\text { performance" }\end{array}$ & $\begin{array}{l}\text { Efeitos } \\
\text { percebidos }\end{array}$ & $\begin{array}{l}\text { Efeitos? Não } \\
\text { existe }\end{array}$ & Efeitos percebidos \\
\hline Estratégia & $\begin{array}{l}\text { Adaptativo } \\
\text { Reactivo / } \\
\text { solução ad hoc }\end{array}$ & $\begin{array}{l}\text { Não adaptativo/ } \\
\text { Proactivo } \\
\text { Controle }\end{array}$ & $\begin{array}{l}\text { Adaptativo } \\
\text { Reactivo / solução ad hoc. }\end{array}$ \\
\hline Conflito & $\begin{array}{l}\text { Sem canais } \\
\text { formais de } \\
\text { expressão de } \\
\text { conflito }\end{array}$ & $\begin{array}{l}\text { Desqualificação do } \\
\text { conflito } \\
\text { Canais formais de } \\
\text { expressão de } \\
\text { conflitos }\end{array}$ & $\begin{array}{l}\text { Sem canais formais de } \\
\text { expressão de conflito. } \\
\text { Centrado na chefia e equipa } \\
\text { e posteriormente na adminis- } \\
\text { tração } \\
\text { GRH primeiro }\end{array}$ \\
\hline Mudança & Experimentam & Não experimentam & Experimentam \\
\hline $\begin{array}{l}\text { Gestão de } \\
\text { pessoas }\end{array}$ & Retenção & Não retenção & Retenção \\
\hline Mercado & Personalizado & Impessoal & Impessoal \\
\hline $\begin{array}{l}\text { Consistência } \\
\text { interna de } \\
\text { práticas de GRH }\end{array}$ & Consistente & $\begin{array}{l}\text { Não consistente } \\
\text { Carreiras técnicas } \\
\text { vs consultoria }\end{array}$ & Consistente \\
\hline $\begin{array}{l}\text { Práticas de } \\
\text { elevado } \\
\text { envolvimento }\end{array}$ & $\begin{array}{l}\text { Formais na } \\
\text { existência, } \\
\text { processo de } \\
\text { desenvolvimento } \\
\text { gradual e } \\
\text { consistente, } \\
\text { negociado }\end{array}$ & $\begin{array}{l}\text { Formais na } \\
\text { existência, } \\
\text { processo de } \\
\text { introdução em } \\
\text { bloco, não nego- } \\
\text { ciado }\end{array}$ & $\begin{array}{l}\text { Formalização na existência, } \\
\text { processo de desenvolvimento } \\
\text { gradual mas pouco consis- } \\
\text { tente } \\
\text { Reduzida sofisticação } \\
\text { intencional das práticas, valo- } \\
\text { rização dos procedimentos }\end{array}$ \\
\hline
\end{tabular}




\begin{tabular}{llll}
\hline Rapidez de & $\begin{array}{l}\text { Ciclos consis- } \\
\text { tentes }\end{array}$ & $\begin{array}{l}\text { Desfasamento de } \\
\text { ciclos. Devido ao } \\
\text { Com tempo de } \\
\text { seu tempo de vida, }\end{array}$ & $\begin{array}{l}\text { Ciclos consistentes, } \\
\text { considerando o tempo }\end{array}$ \\
vida suficiente & $\begin{array}{l}\text { impacto do ciclo } \\
\text { Produção }\end{array}$ & $\begin{array}{l}\text { que permite a } \\
\text { observação do } \\
\text { impacto dos dois } \\
\text { ciclos }\end{array}$ & \\
& RH & & \\
\hline
\end{tabular}

\section{Conclusões}

Estes resultados sugerem que (1) práticas de GRH formalmente similares podem produzir resultados sociais diferentes; (2) o envolvimento, compreensão e aceitação das práticas de GRH são dimensões relevantes de um processo desenvolvimental; (3) práticas que visem o envolvimento são importantes elementos do processo através do qual a GRH tem impacto na performance organizacional e; (4) o grau de especialização e a coerência do sistema interno de GRH com a estratégia da organização contribui para a percepção de utilidade da função RH.

A constatação de que práticas formalmente semelhantes podem produzir resultados diferentes não é novidade absoluta. No entanto, ela chama a atenção para a utilidade de métodos mais qualitativos que permitam a exploração de processos sociais e a consideração de dimensões mais intangíveis destes processos.

Uma destas dimensões, que ficou aparente nesta investigação, relaciona-se com a capacidade de interpretação dos sistemas de práticas de GRH por parte dos colaboradores na organização. A interpretação destas práticas, possível através de processos de envolvimento e comunicação, não ocorre de forma repentina nem puramente individual, mas sim através de um processo continuado de interacção entre os agentes e entre estes e os sistemas de práticas, em que estas interpretações vão sendo validadas e desenvolvidas.

Consequentemente, a possibilidade de interacção entre os diferentes agentes envolvidos é fundamental para que este processo ocorra.

Desenvolvendo um pouco mais esta interpretação dos casos, para além da possibilidade de interacção entre agentes organizacionais e entre estes e as práticas de GRH, um outro factor a levar em consideração é o da legitimidade desta interacção, questão que, por sua vez, se prende com o exercício do poder organizacional. É esta legitimação do espaço de interacção através dos mecanismos de poder que torna a questão da coerência das 
práticas de GRH com a estratégia organizacional uma questão tão central na nossa interpretação destes casos: se a mensagem é que a GRH contribui para a estratégia organizacional, então a interacção sobre e com as práticas de GRH, a possibilidade de contribuir para as modificar e aperfeiçoar deixa de ser apenas ruído ou uma questão secundária para fazer parte das questões importantes da organização. A possibilidade interessante nesta perspectiva é que o efeito das práticas de gestão de recursos humanos tem lugar precisamente através desta interacção e das interpretações que dela resultam e não tanto através de supostos efeitos directos desta ou daquela prática sobre os comportamentos dos agentes organizacionais.

Assim, estes estudos de caso contribuem para o conhecimento do(s) processo(s) pelos quais a GRH tem impacto na organização, evidenciando o papel primordial que o envolvimento e compromisso dos colaboradores, face à organização, têm, em termos dos resultados da organização.

Mais do que a discussão sobre quais as práticas de GRH a adoptar, a harmonização interna dos sistemas de GRH, bem como a sua harmonização com a estratégia da organização, parecem ser campos importantes a explorar.

\section{Referências}

Bamberger, P., Bacharach, S. \& Dyer, L. (1989). Human resources management and organizational effectiveness: high technological entrepreneurial startup firms in Israel. Human Resources Management, 28, 349-366.

Becker, B., \& Gerhart, B. (1996). The impact of human resource management on organisational performance: progress and prospects. Academy of Management Journal, 39, 779-793.

Becker, B. E., \& Huselid, M. A. (1999). Overview: strategic human resource management in five leading firms. Human Resource Management, 38, 287-301 .

Bélanger, J. (2000). The influence of employee involvement on productivity: a review of research. Applied Research Branch Strategic Policy Human Resources Development. Canada, http://www.hrdc-drhc.gc.ca/arb.

Cardon, M. S., \& Stevens, C. E. (2004). Managing human resources in small organizations: What we know? Human Resource Management Review, 14, 295-323.

Cassell, C., Nadin, S., Gray, M., \& Clegg, C. (2002). Exploring human resource management practices in small and medium sized enterprises. Personnel Review, 31, 671-692.

Collins, C., Smith, K. G., \& Stevens, C. K. (2002). Human resource practices, knowledge-creation capability and performance in high technology firms. CAHRS - Center for advanced human resource studies, Working paper series 01-02, pp. 1-36, http://www.ilr.cornell.edu/cahrs. 
Delaney, J. T., \& Huselid, M. A. (1996). The impact of human resource management practices on perceptions of organisational performance. Academy of Management Journal, 39, 949-970.

Delery, J. E., \& Doty, D. H. (1996). Modes of theorising in strategic human resource management: tests of universalistic, contingency, and configurational performance predictions. Academy of Management Journal, 39, 802-825.

Ferris, G. R., Arthur, M. M., Berkson, H. M., Kaplan, D. M., Harrell-Cook, G., \& Frink, D. D. (1998). Toward social context theory of the human resource management - organization effectiveness relationship. Human Resources Management Review, 8, 235-255.

Guest, D. E. (1987). Human resource management and industrial relations. Journal of Management Studies, 24, 503-521.

Guest, D. E. (1999). Human resource management - the workers verdict. Human Resource Management Journal, 9, 5-25.

Guest, D. E. (2001). Human resource management: when research confronts theory. International Journal of Human Resource Management, 12, 1092-1106.

Godard, J., \& Delaney, J. T. (2000). Reflections on the high performance paradigm's implications for industrial relations as a field. Industrial \& Labor Relations Review, 53, 482, 21.

Heneman, R. L., Tansky, J. W. \& Camp, S. M. (2000). Human resource management practices in small and medium-sized enterprises: unanswered questions and future research perspectives. Entrepreneurship, Theory and Practice, 11-26 .

Huselid, M. A (1995). The impact of human resource management practices on turnover, productivity, and corporate financial performance. Academy of Management Journal, 38, 635-672.

Keating, J., Silva, I. S., \& Almeida, H. L. (1999). Gestão de recursos humanos em pequenas e médias empresas. Estudo de um caso. Psicologia, 13, 237-255.

King, N. (1998). Template analysis. G. Symon \& C. Cassell (Eds.) Qualitative methods and analysis in organizational research: a practical guide (pp. 118-134). London: Sage.

Kotey, B., \& Slade, P. (2005). Formal human resource management practices in small growing firms. Journal of Small Business Management, 43, 16-40.

Legge, K. (1995). Human resource management. Rhetorics and realities. England: MacMillan Business.

MacDuffie, J. P. (1995). Human resource bundles and manufacturing performance: organizational logic and flexible production systems in the world auto industry. Industrial \& Labor Relations Review, 48, 197-227.

Marchington, M., \& Grugulis, I. (2000). "Best practice" human resource management: perfect opportunity or dangerous illusion? The International Journal of Human Resource Management, 11, 1104-1124.

Pfeffer, J. (1994). Competitive advantage through people. Boston, MA: Harvard Business School Press. 
Richard, O. C., \& Johnson, N. B. (2001). Understanding the impact of human resource diversity practices on firm perform. Journal of Managerial Issues, 13, 177-195.

Rowden, R. W. (1995). The role of human resource development in the successful small to mid-sized manufacturing businesses: a comparative case study. Human Resource Development Quarterly, vol. 6 (4), winter, pp. 355-373.

Yin, R. K. (1994). Case study. Research, design and methods. London: Sage.

Youndt, M. A. (1996). Human resource management, manufacturing strategy, and firm performance. Academy of Management Journal, 39, 836-867.

Wall, T. D., \& Wood, S. J. (2005). The romance of human resource management and business performance, and the case for big science. Human Relations, 58, 429-462

Wood, S. J. (1999). Human resource management and performance. International Journal of Management Reviews, 1, 367, 472.

Wright, P., \& Gardner, T. M. (2000). Theoretical and empirical challenges in studying: the HR practice-firm performance relationship. CAHRS_Center for advanced human resource studies. Working paper series 00-04, http://www.ilr.cornell.edu/cahrs. 